OPEN ACCESS

Edited by:

Panagiotis Madesis,

University of Thessaly, Greece

Reviewed by:

David T. Stuart,

University of Alberta, Canada

Dae-Hee Lee,

Korea Research Institute of Bioscience and Biotechnology (KRIBB), South

Korea

Mamoru Yamada

Yamaguchi University, Japan

${ }^{*}$ Correspondence:

Yao Yu

yaoyu@fudan.edu.cn

Hong Lu

honglv@fudan.edu.cn

Specialty section: This article was submitted to Industrial Biotechnology, a section of the journal Frontiers in Bioengineering and Biotechnology

Received: 22 October 2021 Accepted: 20 December 2021 Published: 11 January 2022

Citation:

Wu L, Lyu Y, Wu P, Luo T, Zeng J, Shi T, Zhou J, Yu Y and Lu H (2022) Meiosis-Based Laboratory Evolution of the Thermal Tolerance in

Kluyveromyces marxianus.

Front. Bioeng. Biotechnol. 9:799756.

doi: 10.3389/fbioe.2021.799756

\section{Meiosis-Based Laboratory Evolution of the Thermal Tolerance in Kluyveromyces marxianus}

\author{
Li Wu $u^{1,2}$, Yilin Lyu ${ }^{1,2}$, Pingping $W u^{1,2}$, Tongyu Luo ${ }^{1,2}$, Junyuan Zeng ${ }^{1,2}$, Tianfang Shi ${ }^{1,2}$, \\ Jungang Zhou ${ }^{1,2}$, Yao Yu ${ }^{1,2 *}$ and Hong $\mathrm{Lu}^{1,2,3 *}$ \\ ${ }^{1}$ State Key Laboratory of Genetic Engineering, School of Life Sciences, Fudan University, Shanghai, China, ${ }^{2}$ Shanghai \\ Engineering Research Center of Industrial Microorganisms, Shanghai, China, ${ }^{3}$ Shanghai Collaborative Innovation Center for \\ Biomanufacturing Technology, Shanghai, China
}

Kluyveromyces marxianus is the fastest-growing eukaryote and a promising host for producing bioethanol and heterologous proteins. To perform a laboratory evolution of thermal tolerance in K. marxianus, diploid, triploid and tetraploid strains were constructed, respectively. Considering the genetic diversity caused by genetic recombination in meiosis, we established an iterative cycle of "diploid/polyploid meiosis - selection of spores at high temperature" to screen thermotolerant strains. Results showed that the evolution of thermal tolerance in diploid strain was more efficient than that in triploid and tetraploid strains. The thermal tolerance of the progenies of diploid and triploid strains after a two-round screen was significantly improved than that after a one-round screen, while the thermal tolerance of the progenies after the one-round screen was better than that of the initial strain. After a two-round screen, the maximum tolerable temperature of Dip2-8, a progeny of diploid strain, was $3^{\circ} \mathrm{C}$ higher than that of the original strain. Whole-genome sequencing revealed nonsense mutations of PSR1 and PDE2 in the thermotolerant progenies. Deletion of either PSR1 or PDE2 in the original strain improved thermotolerance and two deletions displayed additive effects, suggesting PSR1 and $P D E 2$ negatively regulated the thermotolerance of $K$. marxianus in parallel pathways. Therefore, the iterative cycle of "meiosis - spore screening" developed in this study provides an efficient way to perform the laboratory evolution of heat resistance in yeast.

Keywords: Kluyveromyces marxianus, thermal tolerance, laboratory evolution, meiosis, iterative cycle, PSR1, PDE2

\section{INTRODUCTION}

The mutation is the major driving force of the adaptive evolution, leading to traits in coping with various environmental stress, including heat, salinity, alkalinity, acidity and so on (Wright, 2004). Heat resistance, also called thermal tolerance, is well-recognized as a quantitative trait controlled by a range of genes and pathways (Gao et al., 2016). For example, mutations of Rho1-Pkcl pathway increase the thermal tolerance by affecting the integrity of the cell wall (Huang et al., 2018). Glutathione directly reduces the hydroxyl radical to form $\mathrm{H}_{2} \mathrm{O}$, thereby removing oxygen free radicals in cells, to increase the heat resistance of cells (Grant et al., 1996). Trehalose mainly protects cells from high-temperature damage by stabilizing the cell membrane structure and maintaining the conformation of intracellular proteins (Martinez-Esparza et al., 2011). 
TABLE 1 | Strains used in this study.

\begin{tabular}{|c|c|c|}
\hline Names & Genotypes & Sources \\
\hline FIM1 & & Zhou et al. (2018) \\
\hline $\mathrm{FIM} 1 \Delta u$ & ura3 $\Delta$ & Zhou et al. (2018) \\
\hline $\mathrm{a}-\mathrm{FIM} 1 \Delta u \Delta w$ & MATa ura3 $\Delta \mathrm{hm} / \Delta \operatorname{trp} 1 \Delta$ & This study \\
\hline$\alpha-\mathrm{FIM} 1 \Delta u \Delta h$ & MATa ura3 $\Delta$ hmr $\Delta$ his3 $\Delta$ & This study \\
\hline KM-Diploid-S & $M A T a / a$ ura3 $\Delta$ & This study \\
\hline KM-Diploid & MATa/a ura3 $\Delta \mathrm{hm} / \Delta \mathrm{hmr} \Delta$ & This study \\
\hline KM-Diploid-MATa/a & MATa/a ura3 $\Delta \mathrm{hm} / \Delta \mathrm{hmr} \Delta$ & This study \\
\hline KM-Diploid-MATa/a & MATa/a ura3 $\Delta \mathrm{hm} / \Delta \mathrm{hmr} \Delta$ & This study \\
\hline KM-Triploid & $M A T a / a / a ~ u r a 3 \Delta ~ h m / \Delta h m r \Delta$ & This study \\
\hline KM-Tetraploid & $M A T a / a / a / a$ ura3 $\Delta h m / \Delta h m r \Delta$ & This study \\
\hline FIM1-psr1s & ura3 $\Delta$ psr1 $\Delta$ & This study \\
\hline FIM1-pde2 $\Delta$ & ura3 $\Delta$ pde2 $\Delta$ & This study \\
\hline FIM1-psr1 1 pde2 $\Delta$ & ura3 $\Delta$ psr1 $\Delta$ ped2 $\Delta$ & This study \\
\hline
\end{tabular}

Kluyveromyces marxianus is a food-grade yeast commonly isolated in dairy environments. $K$. marxianus is the fastestgrowing eukaryote reported so far, with a specific growth rate of $0.7-0.99 \mathrm{~h}^{-1}$ (Groeneveld et al., 2009). K. marxianus displays superior thermotolerance, as it can grow at $45^{\circ} \mathrm{C}$ and tolerate temperatures over $50^{\circ} \mathrm{C}$, making it a promising platform for the production of bioethanol and chemicals (Lane and Morrissey, 2010; Gombert et al., 2016). Same as Saccharomyces cerevisiae, $K$. marxianus is a homothallic yeast with two mating types, MATa and MATa. Haploid cells switch the mating type spontaneously and haploid cells are capable of mating with other haploid cells of the opposite mating type to produce MATa/a diploids (Cernak et al., 2018). When encountering adversities unsuitable for growth, diploid cells tend to undergo meiosis to produce tetrad. During meiosis, chromosomal crossover occurs, generating novel allele combinations, and some of which may help cells through harsh environments and subsequently promote the evolution of stress resistance (Owens et al., 2018). By combining meiosis and screen, the researcher successfully increased the ethanol production of S. cerevisiae by $10.96 \%$ (Hou, 2010). In our previous study, a 5-fold increase in the production of ferulic acid lipase was achieved by performing meiosis of $K$. marxianus diploids and subsequent screening of high-yield spores (Wu et al., 2020a). To our knowledge, there is no report of meiosis-mediated evolution of thermal tolerance in yeast yet.

Besides diploid, triploid and tetraploid can also undergo meiosis. In the meiosis of the triploids, homologous chromosomes cannot be distributed equally, resulting in aneuploidy (Charles et al., 2010). In the meiosis of tetraploids, there was an increased chance of asymmetric separation of homologous chromosomes, resulting in progeny cells of multiple ploidies (Loidl, 1995). Therefore, the genetic diversity of spores generated by triploids and tetraploids was expected to be different from that of spores generated by diploids, which might provide a valuable source for the screen of desired phenotypes. However, the triploid and tetraploid strain of $K$. marxianus has not been built in the laboratory yet.

In this study, triploid and tetraploid cells were constructed from diploid K. marxianus cells. Cells were screened by an iterative cycle of "diploid/polyploid - meiosis - selection of spores at high temperature". After a two-round screen, progenies displaying significantly enhanced thermal tolerance were obtained. Whole-genome sequencing suggested PSR1 and PDE2 were negative regulators of the thermotolerance. This study provides the first meiosis-based iterative cycle for the evolution of thermal tolerance in yeast. The method can be applied in the laboratory evolution of resistance to other stresses.

\section{MATERIALS AND METHODS}

\section{Strains and Plasmids}

Strains used in this study were listed in Table 1. Wild-type K. marxianus strain FIM1 was deposited in China General Microbiological Culture Collection Center (CGMCC No.10621). URA3 was deleted in FIM1 to obtain FIM1 $\Delta u$ as described before (Zhou et al., 2018). In FIM1 $\Delta u$, the $H M L$ locus and TRP1 locus were deleted to obtain a-FIM1 $\Delta u \Delta w$, the $H M R$ locus and HIS 3 were deleted to obtain $\alpha$-FIM1 $\Delta u \Delta h, P S R 1$ and PED2 were deleted to obtain FIM1-psr1 $\Delta$ and FIM1-pde2 $\Delta$, respectively. PED2 was deleted in FIM1-psr1 $\Delta$ to obtain FIM1psr $1 \Delta p d e 2 \Delta$. Genes were deleted by CRISPR/Cas 9 as described before (Liu et al., 2018). Primers and plasmids used in the construction were shown in Supplementary Tables S1 and S2.

a-FIM1 $\Delta u \Delta w$ mated with $\alpha$-FIM $1 \Delta u \Delta h$ to form KM-Diploid$\mathrm{S}(M A T \mathrm{a} / \alpha, u r a 3 \Delta)$ as described before (Wu et al., 2020b). The $H M L$ and $H M R$ locus of KM-Diploid-S were deleted by CRISPR/ Cas9 to obtain KM-Diploid. The primer pairs W29F/W29R and W30F/W30R were used to identify the deletion of $H M R$ or $H M L$ locus. The mating type of KM-Diploid (MATa/a) was switched to $M A T a / a$ by replacing MATa locus with MATa using CRISPR/ Cas9, and the resultant strain was KM-Diploid-MATa/a. Similarly, the mating type of KM-Diploid was switched to $M A T \alpha / a$ by replacing MATa locus with MATa, and the resultant strain was KM-Diploid-MATa/a.

To construct the tetraploid strain, a pKD1-based plasmid carrying KanMX6 (pUKDN127-Kan) was transformed into KM-Diploid-MATa/a strain, and a pKD1-based plasmid carrying $h p h M X 4$ (pUKDN127-Hyg) was transformed into KM-Diploid-MATa/a strain. Transformants were cultured separately in YPD plates $(10 \mathrm{~g} / \mathrm{L}$ yeast extract, $20 \mathrm{~g} / \mathrm{L}$ hipolypepton, $20 \mathrm{~g} / \mathrm{L}$ glucose, $20 \mathrm{~g} / \mathrm{L}$ agar) overnight. Cells were mixed and spread onto $\mathrm{ME}$ medium $(50 \mathrm{~g} / \mathrm{L}$ maltose extract, $30 \mathrm{~g} / \mathrm{L}$ agar) to mate. Cells were then cultured at $30^{\circ} \mathrm{C}$ for 2 days and selected on YPD + G418 $(0.2 \mathrm{mg} / \mathrm{ml})$ + Hygromycin $(0.25 \mathrm{mg} / \mathrm{ml})$ plates to obtain tetraploid strain KM-tetraploid. The triploid strain was constructed similarly. KM-Diploid-MATa/a cells transformed with pUKDN127-Kan were mated with a-FIM1 $\Delta u \Delta h$ cells transformed with pUKDN127-Hyg. Triploid strain KM-triploid was selected on the YPD + G418 + Hygromycin plate.

\section{Meiosis and Selection of Spores at High Temperature}

Diploid, triploid or tetraploid cells were cultured in $3 \mathrm{ml} \mathrm{YPD}$ liquid medium (10 g/L yeast extract, $20 \mathrm{~g} / \mathrm{L}$ hipolypepton, $20 \mathrm{~g} / \mathrm{L}$ 
glucose) at $30^{\circ} \mathrm{C}$ for $12 \mathrm{~h}$. Then cultures were inoculated into $50 \mathrm{ml}$ YPA liquid medium (10 g/L potassium acetate, $20 \mathrm{~g} / \mathrm{L}$ hipolypepton, $10 \mathrm{~g} / \mathrm{L}$ yeast extract) at an initial optical density at $600 \mathrm{~nm}\left(\mathrm{OD}_{600}\right)$ of 0.1 , and cultured at $30^{\circ} \mathrm{C}$ for $6-8 \mathrm{~h}$ till $\mathrm{OD}_{600}$ reached 1.0-1.2. Cells were centrifuged and washed twice with sterile water. Cells were resuspended in $50 \mathrm{ml} 2 \%$ potassium acetate (KAc) and cultured at $30^{\circ} \mathrm{C}$ for $24 \mathrm{~h}$ to initiate meiosis and sporulation. $1 \mathrm{ml}$ sample was centrifuged and washed twice with sterile water. The cells were resuspended in $500 \mu \mathrm{l}$ sterile water and then treated with $25 \mu \mathrm{l}$ zymolyase $(5 \mathrm{U} / \mu \mathrm{l}, \mathrm{E} 1004$, Zymoresearch, United States) and $5 \mu \mathrm{l} \beta$-mercaptoethanol at $4^{\circ} \mathrm{C}$ for $24 \mathrm{~h}$. The sample was supplemented with $200 \mu \mathrm{l} 1.5 \%$ NP-40 and incubated at $30^{\circ} \mathrm{C}$ for $30 \mathrm{~min}$ to lyse vegetative cells. Cells were sonicated for $30 \mathrm{~s}$ times (Bioruptor UCD-300, Diagenode, Belgium), and then spread to YPD plates. For the first round of screen, spores generated by KM-Diploid, KMTriploid and $\mathrm{KM}$-tetraploid cells were grown at $43^{\circ} \mathrm{C}$ for 2 days. A total of 392 clones formed on the plates were selected and grown at $43^{\circ} \mathrm{C}$ in $3 \mathrm{ml} \mathrm{YPD}$ liquid medium for $4 \mathrm{~h}$. Then, $3 \mu \mathrm{l}$ culture was spotted onto YPD plates and grown at $43,43,45$, and $46^{\circ} \mathrm{C}$ for 2 days. For the second-round screen, spores generated by diploid $2-2$ and triploid $3-2$ were grown at $46^{\circ} \mathrm{C}$ for 2 days. A total of 580 clones from 2-2 and 680 clones from 3-2 formed on the plates were selected and grown at $46^{\circ} \mathrm{C}$ in $3 \mathrm{ml}$ liquid YPD for $4 \mathrm{~h}$. Then, $3 \mu \mathrm{l}$ culture was spotted onto YPD plates and grown at $45,46,47$, and 48 for 2 days. Substantial growth on the plates indicated thermal tolerance to the temperature.

\section{Determination of Mating Type and Auxotrophic Markers}

The mating types were determined by PCR using three primers (YY270F, YY271F, YY272F). MATa locus produced a band of $1,062 \mathrm{bp}$ and MATa locus produced a band of $1,515 \mathrm{bp}$. Cells failing to grow on the SC-his $(20 \mathrm{~g} / \mathrm{L}$ glucose, $6.7 \mathrm{~g} / \mathrm{L}$ yeast nitrogen base, $40 \mathrm{mg} / \mathrm{L}$ uracil, $40 \mathrm{mg} / \mathrm{L}$ leucine, $40 \mathrm{mg} / \mathrm{L}$ tryptophan, $20 \mathrm{~g} / \mathrm{L}$ agar) and SC-trp plate $(20 \mathrm{~g} / \mathrm{L}$ glucose, $6.7 \mathrm{~g} / \mathrm{L}$ yeast nitrogen base, $40 \mathrm{mg} / \mathrm{L}$ histidine, $40 \mathrm{mg} / \mathrm{L}$ leucine, $40 \mathrm{mg} / \mathrm{L}$ uracil, $20 \mathrm{~g} / \mathrm{L}$ agar) carried $h i s 3 \Delta$ and $\operatorname{trp} 1 \Delta$ auxotrophic markers, respectively. Primers were listed in Supplementary Table S1.

\section{Flow Cytometry}

Cells were grown in YPD overnight. Cells of $800 \mu \mathrm{l}$ cultures were pelleted and washed twice by $1 \mathrm{ml}$ phosphate buffer $(0.2 \mathrm{M}$ $\mathrm{Na}_{2} \mathrm{HPO}_{4}, \mathrm{pH}$ adjusted to 7.0 by $0.1 \mathrm{M}$ citric acid). Cells were resuspended gently in $1 \mathrm{ml}$ of cold $75 \%$ ethanol and stored at $4^{\circ} \mathrm{C}$ for $5 \mathrm{~h}$. Cells were washed by the phosphate buffer once and then resuspended in $800 \mu \mathrm{l}$ phosphate buffer. The sample was supplemented with $10 \mu \mathrm{L}$ RNase $(50 \mathrm{mg} / \mathrm{ml})$ and incubated at $37^{\circ} \mathrm{C}$ for $24 \mathrm{~h}$. Cells were pelleted and resuspended in $1 \mathrm{ml}$ phosphate buffer. Cells were supplemented with $5 \mu \mathrm{l}$ propidium iodide solution $(10 \mu \mathrm{g} / \mathrm{ml}$ propidium iodide in phosphate buffer) and stained for $30 \mathrm{~min}$ in dark. Cells were sonicated for 30 s 3 times. 10,000 cells were measured by a FACS Calibur flow cytometer (Becton Dickinson, United States) and data were analyzed by Flowjo 2.0.

\section{Spot Assay}

For spot assays of FIM1, KM-Diploid, KM-Triploid, KMtetraploid, FIM1-psr1 $\Delta$, FIM1-pde2 $\Delta$ and FIM1-psr1 1 pder2 $\Delta$, cells were grown in $3 \mathrm{ml}$ YPD liquid medium at $30^{\circ} \mathrm{C}$ for $12 \mathrm{~h}$. For the spot assay of spores, individual spore was grown in $3 \mathrm{ml}$ YPD liquid medium and grown at $43^{\circ} \mathrm{C}$ or $46^{\circ} \mathrm{C}$ for $12 \mathrm{~h}$. The culture was adjusted to an $\mathrm{OD}_{600}$ of 0.6 and then diluted fivefold five times. $3 \mu$ ldilutions were spotted on YPD plates. Plates were incubated at $30-48^{\circ} \mathrm{C}$.

\section{Growth Curves}

Cells were grown in $3 \mathrm{ml} \mathrm{YPD} \mathrm{liquid} \mathrm{medium} \mathrm{at} 30^{\circ} \mathrm{C}$ for $12 \mathrm{~h}$ and then diluted into $50 \mathrm{ml}$ fresh YPD liquid medium to start at an $\mathrm{OD}_{600}$ of 0.01 . Cells were grown at $47^{\circ} \mathrm{C}$ for $120 \mathrm{~h}$. The $\mathrm{OD}_{600}$ of the culture was measured every 6 or $12 \mathrm{~h}$. The experiment was performed with three parallel cultures.

\section{Whole-Genome Sequencing}

Cells were grown in $3 \mathrm{ml}$ YPD liquid medium at $30^{\circ} \mathrm{C}$ for $12 \mathrm{~h}$. Genomic DNA was extracted by a Yeast Genomic DNA Extraction kit (D1900, Solarbio, China). Whole-genome sequencing was performed by Illumina Hiseq (Mingma technologies, Shanghai, China). Significant SNPs and InDELs were identified by sequence alignment using the genome of FIM1 as a reference (Yu et al., 2021).

\section{RESULTS}

\section{Improving the Thermal Tolerance of Kluyveromyces marxianus by Meiosis-Based Iterative Screen}

The mating type of $K$. marxianus cells is determined by genes located in the MAT locus. The mating-type switches in $K$. marxianus are expected to occur spontaneously in the same way as reported in Kluyveromyces lactis, during which sequence of $M A T$ locus was replaced by that of $H M L$ locus carrying silent MATa information or by that of $H M R$ locus carrying silent MATa information, in a manner independent of homothallic switching (HO) endonuclease (Barsoum et al., 2010; Lee et al., 2018) (Figure 1A). To prevent the switch of mating type in this study, $H M L$ locus was deleted in MATa cells and $H M R$ locus was deleted in MATa cells to obtain stable MATa $(\mathrm{a}-\mathrm{FIM} 1 \Delta u \Delta w)$ and MATa (a-FIM1 $\Delta u \Delta h)$ haploid cells, respectively (Figure 1B).

a-FIM1 $\Delta u \Delta w$ mated with $\alpha$-FIM1 $\Delta u \Delta h$ to form diploid (KMDiploid-S), in which $H M L$ and $H M R$ loci were deleted subsequently to form stable diploid strain (KM-Diploid). The mating type of KM-Diploid (MATa/a) was switched to MATa/a and $M A T a / \alpha$, respectively. MATa/a cells mated with $M A T \alpha$ and $M A T a / \alpha$ cells, to obtain triploid MATa/a/a and tetraploid MATa/ a/a/a cells, respectively (Figure 1C). Details of the strain construction were described in methods. Ploidies were determined by flow cytometry. DNA contents of haploid, diploid, triploid and tetraploid strains were consistent with theoretical values (Supplementary Figure S1A). 


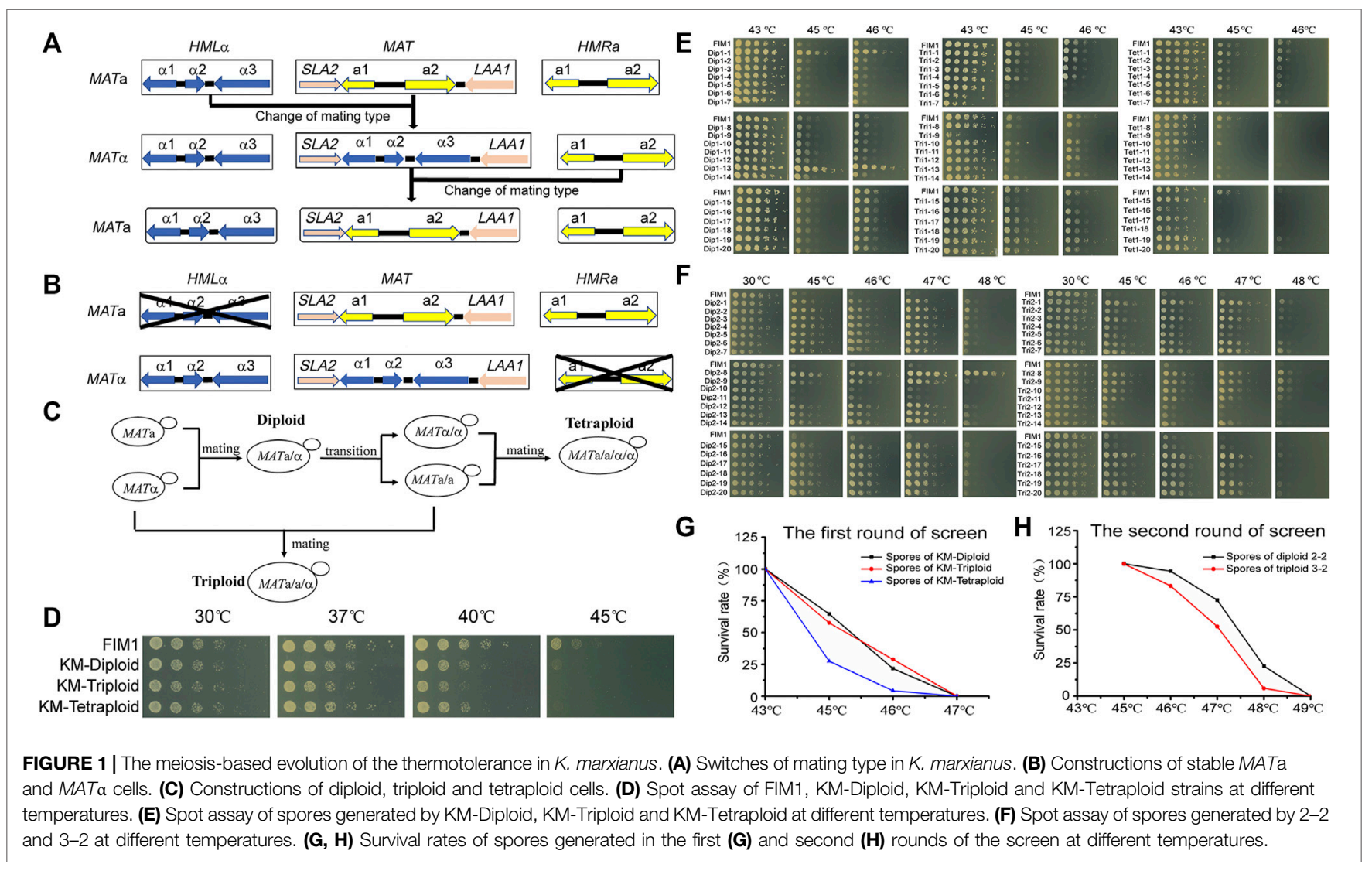

The effect of the ploidy on the thermal tolerance was investigated by a spot assay in the first place. The result showed that ploidy has little effect on cell growth under 30 and $37^{\circ} \mathrm{C}$. The growth of diploid, triploid and tetraploid cells was poorer than that of haploid cells at $40^{\circ} \mathrm{C}$. Haploid cells displayed limited growth at $45^{\circ} \mathrm{C}$, while diploid, triploid and tetraploid cells could not grow at this temperature (Figure 1D). The result suggested, in K. marxianus, cells of higher ploidy levels, including diploid, triploid and tetraploid cells, displayed reduced thermotolerance than haploid cells. A similar result was reported in $\mathrm{S}$. cerevisiae, as the thermotolerance of diploid and tetraploid cells at $50^{\circ} \mathrm{C}$ declined compared to that of haploid cells after a pre-heat shock (Piper et al., 1987).

Diploid, triploid and tetraploid cells underwent meiosis and produced spores in 2\% KAc (Supplementary Figure S1B). Spores were grown at $43^{\circ} \mathrm{C}$ for 2 days till thermotolerant spores formed clones on the plates. A total of 392 clones generated by diploid, triploid or tetraploid were selected and grown at 43,45 , and $46^{\circ} \mathrm{C}$ for 2 days. Less than half of the clones sporulated from diploid, triploid and tetraploid strains could grow at $46^{\circ} \mathrm{C}$ (Supplementary Figure S2). Twenty spores displaying the best thermotolerance in the progenies of diploid, triploid and tetraploid strains were named Dip1-1 20, Tri1-1 20 and Tet1-1 20, respectively. The thermotolerance of these spores was investigated by a spot assay (Figure 1E). Among the spores generated by KM-Diploid, Dip1-1 and Dip1-13 displayed better growth than wild-type FIM1 strain, while the thermal tolerance of the rest 18 spores was similar to that of the FIM1 strain. In the spores generated by KM-Triploid, Tri1-19 displayed better thermal tolerance than FIM1. Five spores, including Tri1-5, 6, 7, 9, 15 displayed worse thermal tolerance than FIM1, while the rest spores were similar to FIM1. In the spores generated by KM-Tetraploid, only Tet1-1 and Tet1-19 displayed similar thermal tolerance as FIM1, while the thermal tolerance of the rest clones was worse than that of FIM1. Results suggested that in the first round of "meiosis - selection of spores at high temperature", the frequency of producing thermotolerant spores from the diploid strain was higher than that from triploid and tetraploid strains.

To find compatible pairs to construct diploid and polyploid strains in the second round of screen, the auxotrophic markers $(\Delta \operatorname{trp} 1$ and $\Delta h i s 3)$, the mating types and ploidies of spores were investigated (Supplementary Figure S3). To evaluate the effect of ploidy on the efficiency of evolution, spores generated by the diploid in the first round of screen were selected to construct the initial diploid strain for the second round of screen. Similarly, spores generated by the triploid were selected to construct triploid, and those by tetraploid were used to construct tetraploid. Based on the above considerations, Dip1-1(MATa, ura3 $\Delta$ ) was selected to mate with Dip1-13 (MATa, ura3 $\Delta$ his3 $\Delta$ ) to form diploid 2-2 (MATa/a, ura3 $\Delta$ ). Tril-19 (MATa, ura3 $\Delta$ ) mated with Tri1-5 (MATa/a, ura3 $\Delta$ ) to form triploid 3-2 (MATa/ $\mathrm{a} / \mathrm{a}$, ura $3 \Delta)$. Tet1-1 (MATa/a, ura3 $\Delta)$ mated with Tet1-15

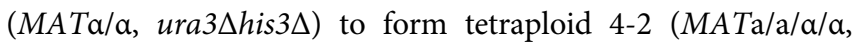



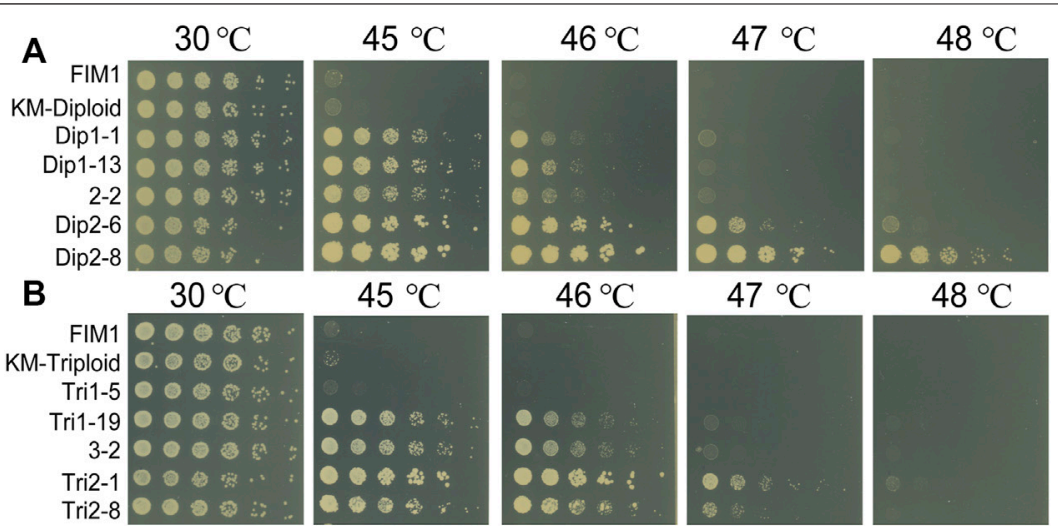

$45^{\circ} \mathrm{C}$

$46^{\circ} \mathrm{C}$
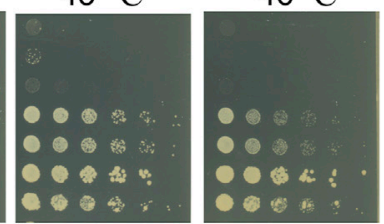

$47^{\circ} \mathrm{C}$

$48^{\circ} \mathrm{C}$

C

E
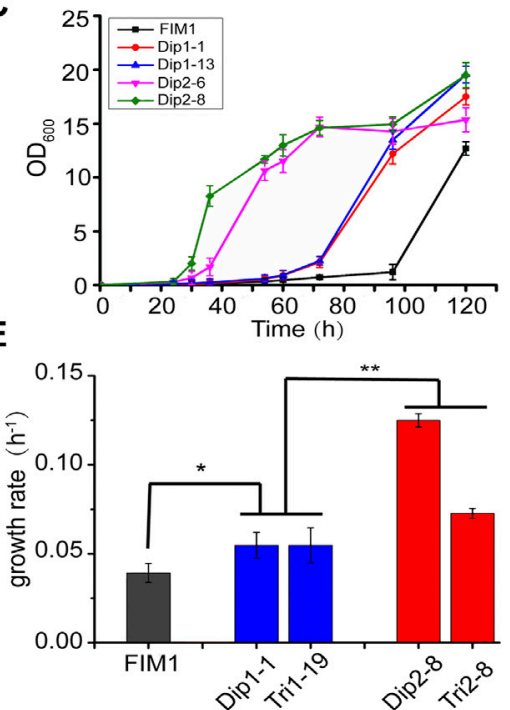

D
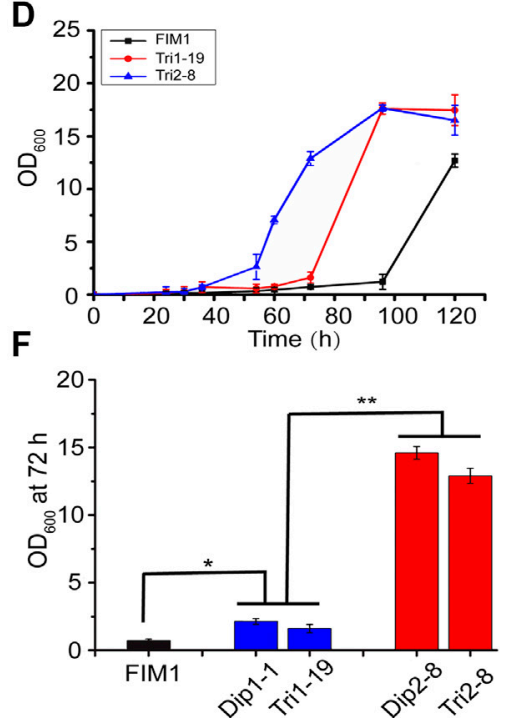

FIGURE 2 | Comparison of the thermotolerant spores generated by diploid and triploid strains. (A, B) Spot assays of the thermotolerant spores generated by diploid strains (A) and triploid strains (B). (C, D) The growth curves of the thermotolerant spores generated by diploid (C) and triploid strains (D) at $47^{\circ} \mathrm{C}$. Values here and below represented mean $\pm \mathrm{SD}(\mathrm{n}=3)$. (E) The maximum growth rate of representative spores generated by diploid and triploid strains at $47^{\circ} \mathrm{C}$. (F) $\mathrm{OD}_{600}$ of representative spores at $72 \mathrm{~h}$. The significant difference was measured by a Student's $t$-test. ${ }^{*} p<0.05,{ }^{\star *} p<0.01$.

ura $3 \Delta)$. The flow cytometry analysis results showed that the DNA contents of 2-2, 3-2 and 4-2 were consistent with their expected ploidies (Supplementary Figure S4A).

In the second round of screen, 2-2, 3-2 and 4-2 were cultured in $2 \%$ KAc to produce spores (Supplementary Figure S4B). First, a portion of spores was grown at $43,45,46$, and $47^{\circ} \mathrm{C}$ for 2 days. Spores of 2-2 and 3-2 could grow at $46^{\circ} \mathrm{C}$, but no spore of $4-2$ could grow at $43^{\circ} \mathrm{C}$. Therefore, $46^{\circ} \mathrm{C}$ was chosen as the temperature for selection in the second round, which was $3^{\circ} \mathrm{C}$ higher than that of the first round. Then, more spores of 2-2 and 3-2 were grown at $46^{\circ} \mathrm{C}$ for 2 days till thermotolerant spores formed clones on the plates. A total of 580 clones generated by $2-2$ and 680 clones generated by 3-2 were selected. Clones were grown at $30,45,46,47$, and $48^{\circ} \mathrm{C}$. The number of highly thermotolerant spores generated by diploid 2-2, which were able to grow at $47^{\circ} \mathrm{C}$ or above, was much higher than by triploid 3-2 (Supplementary Figure S5). Twenty spores of 2-2 displaying the best thermotolerance at $48^{\circ} \mathrm{C}$ were named Dip2$1 \sim 20$ and those of 3-2 were named Tri2-1 20. The thermal tolerance of these spores was investigated by a spot assay (Figure 1F). Dip2-1 20 and Tri2-1 20 exhibited the same growth as FIM1 at $30^{\circ} \mathrm{C}$, while displaying better thermotolerance than FIM1 at higher temperatures. Results indicated that the frequency to obtain thermotolerant spores in the second round of screen was higher than that in the first round of screen. Meanwhile, the highest temperature allowed for growth in the second round of screen, as shown by Dip2-8, was $2^{\circ} \mathrm{C}$ higher than that in the first round of screen. The results suggested that an iterative screen based on the meiosis of diploid and triploid efficiently promoted the evolution of thermal tolerance.

The survival rates of spores growing at different temperatures were compared. In the first round of screen, the survival rates of spores produced by KM-Diploid, KM-Triploid and KMTetraploid at $45^{\circ} \mathrm{C}$ were $64.6,57.5$, and $27.6 \%$, respectively, while those at $46^{\circ} \mathrm{C}$ were $21.8,28.9$, and $4.2 \%$, respectively (Figure 1G). In the second round of screen, the survival rates of spores produced by diploid $2-2$ and triploid $3-2$ at $46^{\circ} \mathrm{C}$ were 
TABLE 2 | SNPs and InDels in thermotolerant spores.

\begin{tabular}{|c|c|c|c|c|c|c|c|}
\hline Posi-tion ${ }^{a}$ & Chromo-some & Mutation sites & ORFs & Change in nucleotide(s) & Change of amino acid & Strain & $\begin{array}{c}\text { Origin } \\
\text { of the mutation }^{b}\end{array}$ \\
\hline \multirow[t]{7}{*}{ CDS } & 2 & 872630 & MUC1 & T1942G & Ser648 > Ala & Dip1-1 & Dip1-1 \\
\hline & 3 & 487709 & PSR1 & G256T & Glu86 > Stop & Dip2-6 & Dip2-6 \\
\hline & 3 & 1272805 & PDE2 & C1019A & Ser340 > Stop & Dip2-6 & Dip2-6 \\
\hline & 3 & 487709 & PSR1 & G256T & Glu86 > Stop & Dip2-8 & Dip2-8 \\
\hline & 3 & 1273144 & PDE2 & G680A & Trp227 > Stop & Dip2-8 & Dip2-8 \\
\hline & 3 & 487943 & PSR1 & +TAAAGAGG & Frameshift & Tri1-19 & Tri1-19 \\
\hline & 3 & 487943 & PSR1 & +TAAAGAGG & Frameshift & Tri2-8 & Tri1-19 \\
\hline \multirow[t]{17}{*}{ NCDS } & 7 & 357288 & - & $+\mathrm{C}$ & - & Dip1-1 & KM-Diploid \\
\hline & 3 & 1572771 & - & $+\mathrm{T}$ & - & Dip1-13 & Dip1-13 \\
\hline & 7 & 357288 & - & $+\mathrm{C}$ & - & Dip1-13 & KM-Diploid \\
\hline & 1 & 1240060 & - & C- $>$ A & - & Dip2-6 & Dip2-6 \\
\hline & 8 & 620828 & - & $\mathrm{T}->\mathrm{G}$ & - & Dip2-6 & Dip2-6 \\
\hline & 3 & 1572771 & - & $+\mathrm{T}$ & - & Dip2-6 & Dip1-13 \\
\hline & 7 & 357288 & - & $+\mathrm{C}$ & - & Dip2-6 & KM-Diploid \\
\hline & 1 & 483871 & - & G- $>$ A & - & Dip2-8 & Dip2-8 \\
\hline & 1 & 541343 & - & G- $>A$ & - & Dip2-8 & Dip2-8 \\
\hline & 1 & 1182783 & - & $A->T$ & - & Dip2-8 & Dip2-8 \\
\hline & 1 & 1240060 & - & $\mathrm{C}->\mathrm{A}$ & - & Dip2-8 & Dip2-8 \\
\hline & 3 & 631197 & - & G- $>$ A & - & Dip2-8 & Dip2-8 \\
\hline & 7 & 357288 & - & $+\mathrm{C}$ & - & Dip2-8 & KM-Diploid \\
\hline & 1 & 204786 & - & $+\mathrm{A}$ & - & Tri1-19 & Tri1-19 \\
\hline & 3 & 1572771 & - & $+\mathrm{T}$ & - & Tri1-19 & Tri1-19 \\
\hline & 7 & 357288 & - & $+\mathrm{C}$ & - & Tri1-19 & KM-Diploid \\
\hline & 7 & 357288 & - & $+\mathrm{C}$ & - & Tri2-8 & KM-Diploid \\
\hline
\end{tabular}

${ }^{a} C D S$, was short for coding sequence and NCDS, was for non-coding sequence.

${ }^{b}$ Name of the ancestor strain from which the mutation was inherited was in bold.

94.3 and $83.3 \%$ respectively, those at $47^{\circ} \mathrm{C}$ were 72.4 and $52.7 \%$, respectively, and those at $48^{\circ} \mathrm{C}$ were 22.6 and $5.9 \%$, respectively (Figure 1H). In general, spores of diploid strain were more thermotolerant than those of triploid and tetraploid strains. The result suggested that the thermotolerance evolved faster in the meiosis of diploid strain than of triploid or tetraploid strain.

\section{The Thermal Tolerance of Spores Generated by a Two-Round Screen was Better Than by a One-Round Screen.}

In the first round of screen, Dip1-1/Dip1-13 and Tri1-19 were the most thermotolerant spores generated by KM-Diploid and KMTriploid, respectively. In the second round of screen, Dip2-8 and Tri2-1/Tri2-8 were the most thermotolerant spores produced by diploid 2-2 and triploid 3-2, respectively. The thermal tolerance of these spores was compared in spot assays (Figures 2A,B). Compared with the wild-type strain FIM1, the highest temperatures allowed for the growth of Dip1-13 and Tri1-19 were $46^{\circ} \mathrm{C}$, that of Dip2-8 was $48^{\circ} \mathrm{C}$, and those of Tri2-1 and Tri28 were $47^{\circ} \mathrm{C}$. The results showed that Dip2-8 was the most thermotolerant spore, which was obtained in the two-round screen.

The growth curves of thermotolerant spores at $47^{\circ} \mathrm{C}$ were investigated. The lag phases of FIM1, Dip1-1, Dip1-13, Dip2-6 and Dip2-8 were 96, 60, 60, 34, $26 \mathrm{~h}$, respectively (Figure 2C). The lag phases of Tri1-19 and Tri2-8 were 70 and $40 \mathrm{~h}$, respectively (Figure 2D). The results suggested spores obtained in the second round of screen displayed shorter lag phases than in the first round screen. The maximum growth rates of spores from the second round of screen (Dip2-8 and Tri2-8) were significantly higher than those of spores from the first round of screen (Dip1-1 and Tri1-19), while maximum growth rates of first-round spores were significantly higher than that of FIM1 (Figure 2E). Similarly, values of $\mathrm{OD}_{600}$, which were indicators of the biomass, of the second-round spores at $72 \mathrm{~h}$ were significantly higher than those of the first-round spores, while values of $\mathrm{OD}_{600}$ of the first-round spores at $72 \mathrm{~h}$ were significantly higher than that of FIM1 (Figure 2F). The shorter the lag phase, the higher the growth rate and the higher biomass at the high temperature indicated the better thermal tolerance. Therefore, through the iterative cycle of "diploid/polyploid -meiosis -selection of spores at high temperature", the thermal tolerance of spores generated by the two-round screen was significantly better than by the oneround screen, while the thermal tolerance of spores generated by the one-round screen was better than that of the original strain.

\section{PSR1 and PDE2 Negatively Regulated the Thermotolerance of $K$. marxianus}

To investigate the mutations underlying the thermal tolerance, Dip1-1, Dip1-13, Dip2-6, Dip2-8, Tri1-19 and Tri2-8 were subjected to the whole-genome sequencing. Seven mutations inside coding sequences, ten single-nucleotide polymorphisms (SNPs) and three insertion-deletions (InDels) inside non-coding sequences (NCDS) were identified in the spores (Table 2). Origins of some mutations could be traced back to ancestor strains (Table 2). For example, a frameshift in PSR1 in Tri2-8 was 


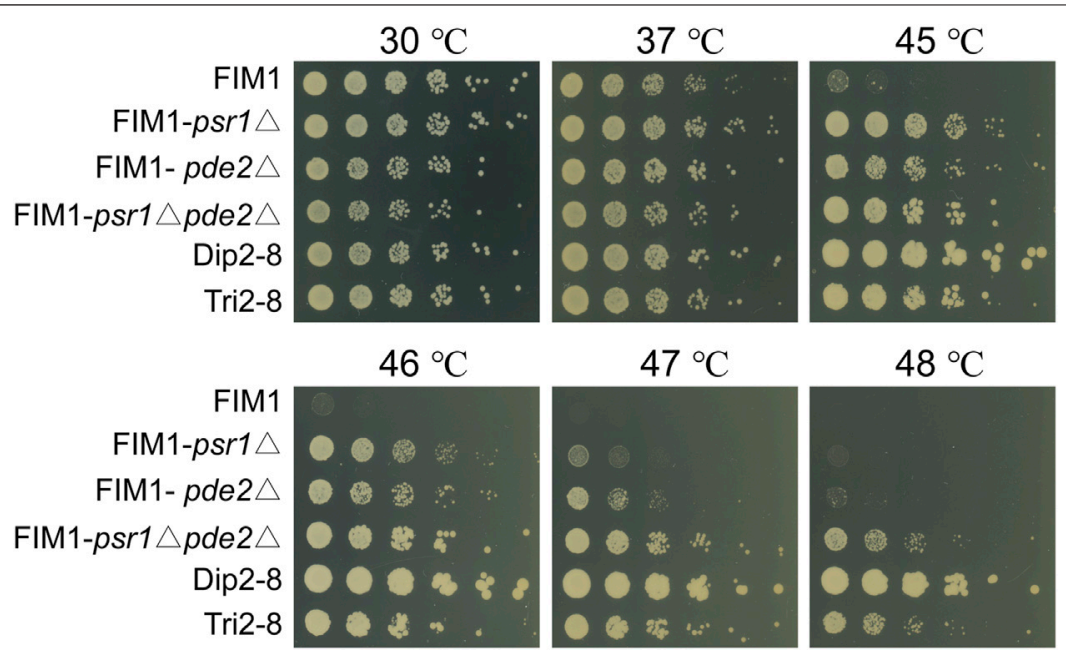

FIGURE 3 | Spot assays of FIM1 carrying the deletion of PSR1, PDE2 or a combination of both deletions. FIM1, Dip2-8 and Tri2-8 were spotted as controls.

inherited from Tri1-19. An insertion of "C" at 357288bp of chromosome 7 in all six spores was presumably inherited from KM-Diploid. Other SNPs and InDels might be caused by replication and repair errors during the meiosis or the vegetative growth afterwards. Notably, compared with the wild-type strain FIM1, Dip2-6 and Dip2-8 contained nonsense mutations of PSR1 and PDE2 genes, while Tri1-19 and Tri2-8 contained frameshift of PSR1. The result suggested mutations of PSR1 and PDE2 were the major contributors to the improved thermotolerance. To validate this idea, PSR1 and PDE2 were deleted individually or together in FIM1 to construct FIM1-psr1 $\Delta$, FIM1-pde2 $\Delta$ and FIM1-psr $1 \Delta p d e 2 \Delta$ strains, respectively (Supplementary Figure S6). These strains, along with Dip2-8, Tri2-8 and FIM1, were grown at $30^{\circ} \mathrm{C}, 37^{\circ} \mathrm{C}, 45^{\circ} \mathrm{C}, 46^{\circ} \mathrm{C}, 47^{\circ} \mathrm{C}$, and $48^{\circ} \mathrm{C}$. No significant growth difference was found between strains at $30^{\circ} \mathrm{C}$ and $37^{\circ} \mathrm{C}$. The growth of FIM1-psr1s and FIM1-pde2 $\Delta$ was significantly better than that of FIM1 at $45^{\circ} \mathrm{C}, 46^{\circ} \mathrm{C}$ and $47^{\circ} \mathrm{C}$. The growth of FIM1-psr1 $\Delta$ pde2 $\Delta$ was better than that of FIM1-psr1 $\Delta$ and FIM1pde $2 \Delta$ at 45,46 , and $47^{\circ} \mathrm{C}$ (Figure 3). These results suggested that PSR1 and PDE2 were key genes that negatively regulated the thermotolerance in $K$. marxianus and that two genes functioned in parallel pathways. However, the growth of FIM1-psr $1 \Delta$ pde2 $\Delta$ at $47^{\circ} \mathrm{C}$ and $48^{\circ} \mathrm{C}$ was poorer than that of Dip2-8, which contained five SNP and one InDel inside NCDS, besides the mutation of PSR1 and PDE2 (Table 2). The growth FIM1-psr $1 \triangle$ at $47^{\circ} \mathrm{C}$ and $48^{\circ} \mathrm{C}$ was poorer than that of Tri2-8, which contained one InDel inside NCDS, besides the mutation of PSR1 (Table 2). The result suggested that mutations inside NCDS, other than the mutations of PSR1 and PDE2, also contributed to the thermotolerance.

\section{DISCUSSION}

High yielding of heterologous proteins, including $\beta$-glucosidase (Su et al., 2021), feruloyl esterase (Liu et al., 2018), and virus-like particles (Duan et al., 2019), has been successfully achieved in $K$. marxianus. Meanwhile, superior features of fast growth, thermotolerance and the capacity to assimilate pentose facilitate the production of bioethanol and chemicals in $K$. marxianus (Suzuki et al., 2019). Collectively, K. marxianus is a microbial cell factory with great potentials.

Improving the thermal tolerance of $K$. marxianus is a necessity to promote its industrial applications, especially in the production of ethanol. Based on the genetic diversity produced by the recombination of meiosis, we established an iterative cycle of "diploid/polyploid - meiosis - selection of spores at high temperature" to improve the thermal tolerance of $K$. marxianus. During the vegetative growth of yeast cells, the genome-wide single-nucleotide mutation rate was at $2-3 \times$ $10^{-10}$ per base per generation (Lynch et al., 2008; Zhu et al., 2014), and continuous culturing yeast cells at high temperature were expected to enrich mutations that improved thermal tolerance of cells. Consistent with this idea, the growth rate of S. cerevisiae at $40^{\circ} \mathrm{C}$ was increased by 1.57 times, through the continuous passage of the culture at $39.5^{\circ} \mathrm{C}$ for more than 90 days (Caspeta et al., 2014). During meiosis, DNA repair associated recombination was mutagenic, providing extra genetic diversity that can contribute to adaptive evolution (Rattray et al., 2015). In this study, we established an iterative cycle of "diploid/polyploid meiosis - selection of spores at high temperature" in $K$. marxianus, and successfully improved the maximum temperature allowed for growth by $3^{\circ} \mathrm{C}$ after only two rounds of the screen. Furthermore, the average concentration of ethanol produced by second-round spores (Dip 2-8 and Tri2-8) at $45^{\circ} \mathrm{C}$ was significantly more than that by one-round spores (Dip1-1 and Tri1-19) (Supplementary Figure S7). The result suggested the ethanol productivity at high temperatures was also improved during the iterative cycle of evolution.

Compared with the evolution of vegetatively growing cells, one obstacle to the meiosis-based evolution was the requirement of constructing diploid/polyploid strains for each round of screen. In some cases, spores displaying better thermotolerance could not be selected to construct diploid/polyploid strains because mating types of spores were not compatible. For example, Tri1-4 (MATa/ 
a) was more thermotolerant than Tri1-5 (MATa/a), but the latter was selected to construct a triploid strain as it was compatible with Tri1-19 (MATa) (Figure 1E, Supplementary Figure S3). Therefore, to construct diploid/triploid from the most thermotolerant spores, a convenient protocol to switch the mating type needed to be established in the following study. In some cases, the mating type was not consistent with the ploidy. For example, the mating type of Dip1-2 was identified as MATa/a by PCR (Supplementary Figure S3). However, Dip1-2 was identified to be a haploid strain in the flow cytometry analysis. Meiosis might result in rearrangement at the MAT locus and lead to the production of multiple MAT loci in the genome.

In this study, diploid, triploid and tetraploid strains were selected for the iterative screen. The proportion of thermotolerant spores from the diploid was higher than that from the triploid, and that from the triploid was higher than that from the tetraploid (Figures 1E,F). This result might be related to the fact that the thermal tolerance of K. marxianus decreased with increased ploidies (Figure 1D). The same results were reported for S. cerevisiae (Zhang et al., 2017). In addition, aneuploidy spores generated by the triploid strain might lead to an imbalance of cellular energy metabolism, which was detrimental to stress resistance (Torres et al., 2007). The growth of aneuploid cells at high temperatures imposed an extra burden on the chromosome segregation and might cause a higher frequency of chromosome rearrangements and loss, which reduced the viability of progenies and efficiency of adaptive evolution (Yona et al., 2012).

Thermotolerant spores obtained in this screen, including Dip1-1, Dip1-13, Dip2-6, Dip2-8, Tri1-19 and Tri2-8, grew as well as wild-type FIM1 at $30^{\circ} \mathrm{C}$. Whole-genome sequencing of these spores obtained in our screen did not reveal any rearrangement or loss of chromosome fragments, suggesting the natural chromosome structures of $K$. marxianus were required for the balance of normal growth at the regular temperature and improved growth at high temperatures. Four out of six thermotolerant spores contained mutations of PSR1, while two spores contained mutations of PDE2. FIM1 cells carrying an individual deletion of PSR1 or PDE2 exhibited increased thermotolerance, while deletion of both genes displayed additive effects on the thermotolerance. The result indicated that PSR1 and PDE2 negatively regulated the growth of $K$. marxianus at high temperatures (up to $48^{\circ} \mathrm{C}$ ) through parallel pathways. In $S$. cerevisiae, PDE2 encodes a highaffinity cAMP phosphodiesterase that catalyzes the degradation of CAMP, and thus negatively regulates numerous cAMP-dependent pathways (Sass et al., 1986). Psr1 is a membrane-associated phosphatase. Psr1 and its partner Psr2 form a complex with Whi2 to negative regulate TORC1, which is a signalling complex regulating the response to nutrients (Kaida et al., 2002; Chen et al., 2018). Notably, deletion of PDE2 and PSR1 reduced the viability of the cells after a transient heat shock, probably through constitutively activated cAMP-dependent pathways and TORC1 pathways, respectively (Jones et al., 2004; Teng et al., 2011). The results indicated PSR1 and PDE2 were positive regulators of the response to heat shock in S. cerevisiae, which looks like contradicting the results in $K$. marxianus. However, the mechanism supporting the survival of short exposure to extremely high temperatures differs that regulating growth at high temperatures. For example, Hsp104 plays a vital role in helping cells survive short-term heat shock, but is not required for the growth at high temperatures (Lindquist and Kim, 1996). Besides, there was no direct proof indicating that PSR1 and PDE2 were required for continuous growth at high temperatures in $S$. cerevisiae. $K$. marxianus is more thermotolerant than $S$. cerevisiae (Fonseca et al., 2008). It is possible that complex networks regulated by PSR1 and PDE2 in K. marxianus, such as PKA and TORC1 pathways, are rewired during the evolution to negatively regulate the growth at high temperatures. The detailed mechanism is worthwhile to be investigated in the following study.

\section{DATA AVAILABILITY STATEMENT}

The original contributions presented in the study are publicly available. This data can be found here: https://www.ncbi.nlm.nih. gov/bioproject/PRJNA732519.

\section{AUTHOR CONTRIBUTIONS}

HL and YY designed the study and supervised the project. LW performed the screen, analyzed the data, and wrote the manuscript. YL and WP assisted the screen. TL and JZe analyzed the sequencing data. TS constructed strains for mating. JZh assisted mating and screen. All authors have revised the manuscript and approved the final version.

\section{FUNDING}

This work was supported by the National Key Research and Development Program of China 2021YFC2100203, Tianjin Synthetic Biotechnology Innovation Capacity Improvement Project TSBICIP-KJGG-006, Science and Technology Research Program of Shanghai 19DZ2282100, and National Natural Science Foundation of China 31770094.

\section{ACKNOWLEDGMENTS}

We thank Qin Lan, Haiyan Ren for their assistance in the experiment of ethanol productivity.

\section{SUPPLEMENTARY MATERIAL}

The Supplementary Material for this article can be found online at: https:/www.frontiersin.org/articles/10.3389/fbioe.2021.799756/ full\#supplementary-material 


\section{REFERENCES}

Barsoum, E., Martinez, P., and Åström, S. U. (2010). a3, a Transposable Element that Promotes Host Sexual Reproduction. Genes Dev. 24 (1), 33-44. doi:10.1101/gad.557310

Caspeta, L., Chen, Y., Ghiaci, P., Feizi, A., Buskov, S., Hallström, B. M., et al. (2014). Altered Sterol Composition Renders Yeast Thermotolerant. Science 346 (6205), 75-78. doi:10.1126/science.1258137

Cernak, P., Estrela, R, Poddar, S., Skerker, J. M., Cheng, Y.-F., Carlson, A. K., et al. (2018). Engineering Kluyveromyces Marxianus as a Robust Synthetic Biology Platform Host. mBio 9 (5), e01410-18. doi:10.1128/mBio.01410-18

Charles, J. S., Hamilton, M. L., and Petes, T. D. (2010). Meiotic Chromosome Segregation in Triploid Strains of Saccharomyces cerevisiae. Genetics 186 (2), 537-550. doi:10.1534/genetics.110.121533

Chen, X., Wang, G., Zhang, Y., Dayhoff-Brannigan, M., Diny, N. L., Zhao, M., et al. (2018). Whi2 Is a Conserved Negative Regulator of TORC1 in Response to Low Amino Acids. Plos Genet. 14 (8), e1007592. doi:10.1371/journal.pgen.1007592

Duan, J., Yang, D., Chen, L., Yu, Y., Zhou, J., and Lu, H. (2019). Efficient Production of Porcine Circovirus Virus-like Particles Using the Nonconventional Yeast Kluyveromyces Marxianus. Appl. Microbiol. Biotechnol. 103 (2), 833-842. doi:10.1007/s00253-018-9487-2

Fonseca, G. G., Heinzle, E., Wittmann, C., and Gombert, A. K. (2008). The Yeast Kluyveromyces Marxianus and its Biotechnological Potential. Appl. Microbiol. Biotechnol. 79 (3), 339-354. doi:10.1007/s00253-008-1458-6

Gao, L., Liu, Y., Sun, H., Li, C., Zhao, Z., and Liu, G. (2016). Advances in Mechanisms and Modifications for Rendering Yeast Thermotolerance. J. Biosci. Bioeng. 121 (6), 599-606. doi:10.1016/j.jbiosc.2015.11.002

Gombert, A. K., Madeira, J. V., Jr., Cerdán, M.-E., and González-Siso, M.-I. (2016). Kluyveromyces Marxianus as a Host for Heterologous Protein Synthesis. Appl. Microbiol. Biotechnol. 100 (14), 6193-6208. doi:10.1007/s00253-016-7645-y

Grant, C. M., MacIver, F. H., and Dawes, I. W. (1996). Glutathione Is an Essential Metabolite Required for Resistance to Oxidative Stress in the yeastSaccharomyces Cerevisiae. Curr. Genet. 29 (6), 511-515. doi:10.1007/BF02426954

Groeneveld, P., Stouthamer, A. H., and Westerhoff, H. V. (2009). Super Life - How and Why 'cell Selection' Leads to the Fastest-Growing Eukaryote. FEBS J. 276 (1), 254-270. doi:10.1111/j.1742-4658.2008.06778.x

Hou, L. (2010). Improved Production of Ethanol by Novel Genome Shuffling in Saccharomyces cerevisiae. Appl. Biochem. Biotechnol. 160 (4), 1084-1093. doi:10.1007/s12010-009-8552-9

Huang, C.-J., Lu, M.-Y., Chang, Y.-W., and Li, W.-H. (2018). Experimental Evolution of Yeast for High-Temperature Tolerance. Mol. Biol. Evol. 35 (8), 1823-1839. doi:10.1093/molbev/msy077

Jones, D. L., Petty, J., Hoyle, D. C., Hayes, A., Oliver, S. G., Riba-Garcia, I., et al. (2004). Genome-Wide Analysis of the Effects of Heat Shock on aSaccharomyces cerevisiaeMutant with a Constitutively Activated cAMP-dependent Pathway. Comp. Funct. Genomics 5 (5), 419-431. doi:10.1002/cfg.415

Kaida, D., Yashiroda, H., Toh-e, A., and Kikuchi, Y. (2002). Yeast Whi2 and Psr1Phosphatase Form a Complex and Regulate STRE-Mediated Gene Expression. Genes Cells 7 (6), 543-552. doi:10.1046/j.1365-2443.2002.00538.x

Lane, M. M., and Morrissey, J. P. (2010). Kluyveromyces Marxianus: A Yeast Emerging from its Sister's Shadow. Fungal Biol. Rev. 24 (1), 17-26. doi:10.1016/j.fbr.2010.01.001

Lee, M.-H., Lin, J.-J., Lin, Y.-J., Chang, J.-J., Ke, H.-M., Fan, W.-L., et al. (2018). Genome-wide Prediction of CRISPR/Cas9 Targets in Kluyveromyces Marxianus and its Application to Obtain a Stable Haploid Strain. Sci. Rep. 8 (1), 7305. doi:10.1038/s41598-018-25366-Z

Lindquist, S., and Kim, G. (1996). Heat-shock Protein 104 Expression Is Sufficient for Thermotolerance in Yeast. Proc. Natl. Acad. Sci. 93 (11), 5301-5306. doi:10.1073/ pnas.93.11.5301

Liu, Y., Mo, W.-J., Shi, T.-F., Wang, M.-Z., Zhou, J.-G., Yu, Y., et al. (2018). Mutational Mtc6p Attenuates Autophagy and Improves Secretory Expression of Heterologous Proteins in Kluyveromyces Marxianus. Microb. Cel Fact 17 (1), 144. doi:10.1186/s12934018-0993-9

Loidl, J. (1995). Meiotic Chromosome Pairing in Triploid and Tetraploid Saccharomyces cerevisiae. Genetics 139 (4), 1511-1520. doi:10.1093/genetics/139.4.1511

Lynch, M., Sung, W., Morris, K., Coffey, N., Landry, C. R., Dopman, E. B., et al. (2008). A Genome-wide View of the Spectrum of Spontaneous Mutations in Yeast. Proc. Natl. Acad. Sci. 105 (27), 9272-9277. doi:10.1073/pnas.0803466105

Martinez-Esparza, M., Tapia-Abellan, A., Vitse-Standaert, A., Garcia-Penarrubia, P., Arguelles, J. C., Poulain, D., et al. (2011). Glycoconjugate Expression on the Cell wall of Tps1/tps1 Trehalose-Deficient Candida Albicans Strain and Implications for its Interaction with Macrophages. Glycobiology 21 (6), 796-805. doi:10.1093/glycob/ cwr007

Owens, S., Tang, S., and Hunter, N. (2018). Monitoring Recombination during Meiosis in Budding Yeast. Methods Enzymol. 601, 275-307. doi:10.1016/bs.mie.2017.12.005

Piper, P. W., Davies, M. W., Curran, B., Lockheart, A., Spalding, A., and Tuite, M. F. (1987). The Influence of Cell Ploidy on the Thermotolerance of Saccharomyces cerevisiae. Curr. Genet. 11 (8), 595-598. doi:10.1007/BF00393921

Rattray, A., Santoyo, G., Shafer, B., and Strathern, J. N. (2015). Elevated Mutation Rate during Meiosis in Saccharomyces cerevisiae. Plos Genet. 11 (1), e1004910. doi:10.1371/ journal.pgen.1004910

Sass, P., Field, J., Nikawa, J., Toda, T., and Wigler, M. (1986). Cloning and Characterization of the High-Affinity cAMP Phosphodiesterase of Saccharomyces cerevisiae. Proc. Natl. Acad. Sci. 83 (24), 9303-9307. doi:10.1073/pnas.83.24.9303

Su, M., Hu, Y., Cui, Y., Wang, Y., Yu, H., Liu, J., et al. (2021). Production of $\beta$-glucosidase from Okara Fermentation Using Kluyveromyces Marxianus. J. Food Sci. Technol. 58 (1), 366-376. doi:10.1007/s13197-020-04550-y

Suzuki, T., Hoshino, T., and Matsushika, A. (2019). High-temperature Ethanol Production by a Series of Recombinant Xylose-Fermenting Kluyveromyces Marxianus Strains. Enzyme Microb. Technol. 129, 109359. doi:10.1016/j.enzmictec.2019.109359

Teng, X., Cheng, W.-C., Qi, B., Yu, T.-X., Ramachandran, K., Boersma, M. D., et al. (2011). Gene-dependent Cell Death in Yeast. Cell Death Dis 2, e188. doi:10.1038/ cddis. 2011.72

Torres, E. M., Sokolsky, T., Tucker, C. M., Chan, L. Y., Boselli, M., Dunham, M. J., et al. (2007). Effects of Aneuploidy on Cellular Physiology and Cell Division in Haploid Yeast. Science 317 (5840), 916-924. doi:10.1126/science.1142210

Wright, B. E. (2004). Stress-directed Adaptive Mutations and Evolution. Mol. Microbiol. 52 (3), 643-650. doi:10.1111/j.1365-2958.2004.04012.x

Wu, L., Wang, M., Zha, G., Zhou, J., Yu, Y., and Lu, H. (2020b). A Protocol of Rapid Laboratory Evolution by Genome Shuffling in Kluyveromyces Marxianus. MethodsX 7, 101138. doi:10.1016/j.mex.2020.101138

Wu, L., Wang, M., Zha, G., Zhou, J., Yu, Y., and Lu, H. (2020a). Improving the Expression of a Heterologous Protein by Genome Shuffling in Kluyveromyces Marxianus. J. Biotechnol. 320, 11-16. doi:10.1016/j.jbiotec.2020.06.007

Yona, A. H., Manor, Y. S., Herbst, R. H., Romano, G. H., Mitchell, A., Kupiec, M., et al. (2012). Chromosomal Duplication Is a Transient Evolutionary Solution to Stress. Proc. Natl. Acad. Sci. 109 (51), 21010-21015. doi:10.1073/pnas.1211150109

Yu, Y., Mo, W., Ren, H., Yang, X., Lu, W., Luo, T., et al. (2021). Comparative Genomic and Transcriptomic Analysis Reveals Specific Features of Gene Regulation in Kluyveromyces Marxianus. Front. Microbiol. 12, 598060. doi:10.3389/fmicb.2021.598060

Zhang, K., Fang, Y.-H., Gao, K.-H., Sui, Y., Zheng, D.-Q., and Wu, X.-C. (2017). Effects of Genome Duplication on Phenotypes and Industrial Applications of Saccharomyces cerevisiae Strains. Appl. Microbiol. Biotechnol. 101 (13), 5405-5414. doi:10.1007/ s00253-017-8284-7

Zhou, J., Zhu, P., Hu, X., Lu, H., and Yu, Y. (2018). Improved Secretory Expression of Lignocellulolytic Enzymes in Kluyveromyces Marxianus by Promoter and Signal Sequence Engineering. Biotechnol. Biofuels 11, 235. doi:10.1186/s13068-018-1232-7

Zhu, Y. O., Siegal, M. L., Hall, D. W., and Petrov, D. A. (2014). Precise Estimates of Mutation Rate and Spectrum in Yeast. Proc. Natl. Acad. Sci. 111 (22), E2310-E2318. doi:10.1073/pnas.1323011111

Conflict of Interest: The authors declare that the research was conducted in the absence of any commercial or financial relationships that could be construed as a potential conflict of interest.

Publisher's Note: All claims expressed in this article are solely those of the authors and do not necessarily represent those of their affiliated organizations, or those of the publisher, the editors and the reviewers. Any product that may be evaluated in this article, or claim that may be made by its manufacturer, is not guaranteed or endorsed by the publisher.

Copyright (C) $2022 \mathrm{Wu}, \mathrm{Lyu}, \mathrm{Wu}, \mathrm{Luo}$, Zeng, Shi, Zhou, Yu and Lu. This is an openaccess article distributed under the terms of the Creative Commons Attribution License (CC BY). The use, distribution or reproduction in other forums is permitted, provided the original author(s) and the copyright owner(s) are credited and that the original publication in this journal is cited, in accordance with accepted academic practice. No use, distribution or reproduction is permitted which does not comply with these terms. 